\title{
Accidental cut of esophageal temperature probe during lobectomy for lung cancer - A case report -
}

\section{Seok Soo Lee ${ }^{1}$, Eun Kyung Choi ${ }^{2}$, Nyeong keon Kwon ${ }^{2}$, Kwang Beom Kim², and Sang-Jin Park ${ }^{2}$}

Departments of ${ }^{1}$ Thoracic and Cardiovascular Surgery, ${ }^{2}$ Anesthesiology and Pain Medicine, Yeungnam University College of Medicine, Daegu, Korea
Received March 22, 2018

Revised June 19, 2018

Accepted July 13, 2018

\section{Corresponding author}

Sang-Jin Park, M.D., Ph.D.

Department of Anesthesiology

and Pain Medicine, Yeungnam

University College of Medicine, 170

Hyeonchung-ro, Nam-gu, Daegu

42415, Korea

Tel: 82-53-620-3366

Fax: 82-53-626-5275

E-mail: apsj0718@naver.com

\section{ORCID}

http://orcid.org/0000-0002-4838-2664

\begin{abstract}
Adequate maintenance of body temperature during general anesthesia is necessary for safety. Generally, esophageal temperature probe is commonly used in practice for measuring core temperature because of its reliability and feasibility. Proper placement of esophageal temperature probe is important to avoid complications. In this case report, we describe our experience with a patient undergoing lobectomy of the lung in whom the esophageal temperature probe that was misplaced into the right intermediate bronchus was accidentally cut. This case highlights the need to carefully assess correct position of the temperature probe, especially in patients undergoing one-lung ventilation.
\end{abstract}

Keywords: Lobectomy; Position; Probe; Temperature.
Adequate maintenance of body temperature during general anesthesia is necessary for patients' safety. Perioperative thermal disturbance can cause numerous adverse outcomes such as wound infection, coagulopathy, delayed postanesthetic recovery, and prolonged hospitalization [1-3]. Core temperature can be accurately monitored at the tympanic membrane, distal esophagus, nasopharynx, and pulmonary artery [4]. Generally, esophageal temperature probe is used because of its reliability [5] and feasibility in practice. Liu et al. [6] suggested that esophageal temperature is more reliable than tympanic temperature during thoracotomy. Proper placement of esophageal temperature probe is important to avoid unwanted complications: inadvertent temperature reading, airway irritation, and serious hypoxemia [7-9].

Herein, we reported a rare complication involving esophageal temperature probe which was misplaced into the right intermediate bronchus and accidentally cut in a patient undergoing lobectomy of the lung.

\section{CASE REPORT}

A 79-year-old man (height $163 \mathrm{~cm}$, body weight $63 \mathrm{~kg}$ ) was scheduled to undergo middle and lower bilobectomy of the right lung through video-assisted thoracic surgery. The patient was diagnosed with adenocarcinoma of the right middle lobe. Chest radiographic examination before surgery revealed a 3-cm sized mass-like lesion at the right middle lobe bronchus with distal segmental atelectasis; pulmonary function test showed normal results; and systemic positron emission computed tomography showed no metastasis except in the right lung.

On entrance into the operation room, patients' intraarterial blood pressure, electrocardiography, pulse oximetry, end-tidal $\mathrm{CO}_{2}$, and central venous pressure were monitored. Temperature of the operation room was set at $25^{\circ} \mathrm{C}$ and breathing circuit was automatically heated and humidified. After induction with propofol and remifentanil through target-controlled infusion, the trachea was intubated with $37 \mathrm{Fr}$

This is an Open Access article distributed under the terms of the Creative Commons Attribution Non-Commercial License (http://creativecommons.org/licenses/by-nc/4.0) which permits unrestricted non-commercial use, distribution, and reproduction in any medium, provided the original work is properly cited.

Copyright (c) the Korean Society of Anesthesiologists, 2018 
double lumen endobronchial tube followed by assessment of proper positioning of the tube through fiberoptic bronchoscopy. The patient's position was changed from supine to left lateral decubitus; subsequently, position of the endobronchial tube was reconfirmed through use of fiberoptic bronchoscope. An esophageal temperature probe (12 Fr probe, Meditop Co., Ltd., Korea) was inserted through the mouth with relative ease despite slight resistance at the oropharynx. Audible heartbeat and lung sound were detected through ear pieces located approximately $30 \mathrm{~cm}$ inside from the upper lip. The sounds were similar to that in other cases with temperature probe placement in the esophagus. At that time, body temperature was $36.2^{\circ} \mathrm{C}$ and there was no change of the peak airway pressure, tidal volume, and oxygen saturation. At mid duration of surgery, when the right intermediate bronchus was cut, the body temperature reading was suddenly interrupted and temperature probe showed no movement which suggested that it was fixed or anchored. After few minutes, little amount of blood was observed in the lumen of the temperature probe following passing of blood through the tracheal lumen of the opened endobronchial tube. Attempt was made to check the bronchus under fiberoptic bronchoscopy, however, it was difficult due to persistent bleeding. The surgeons were informed about the complication, and foreign body was detected within the right bronchus. The foreign body was fixed at the resection margin of the right intermediate bronchus and was identified as a portion of severed temperature probe. Remnant of the temperature probe was found in the removed lobe. It was assumed that the temperature probe, which was inserted into the right intermediate bronchus, was cut and sealed within the bronchus. The bronchus was cut again at a more proximal site so as to remove the incomplete resection margin caused by the temperature probe tip, and the remaining portion of the temperature probe was removed through the trachea. The remaining operation was uneventful and thoracic paravertebral block was performed to reduce postoperative pain at the end of surgery. Total length of the removed temperature probe was not different from that of the intact temperature probe (Fig. 1) and no remnant was detected on acquired chest radiographic images. The patient was extubated and transferred to the intensive care unit. There were no adverse symptoms such as hoarseness after extubation. The patient was discharged uneventfully at 10-days post-surgery.

\section{DISCUSSION}

Central thermoregulatory system is changed in patients under general anesthesia. Vasodilatory properties caused by anesthetic drugs induce core-to-peripheral thermal redistribution, which is the main cause of intraoperative hypothermia. Moreover, anesthetics induce ineffective thermoregulation, and exposure to cold environment in the operating room, and evaporation from surgical sites can cause hypothermia. Therefore, core temperature monitoring should be required in patients undergoing general anesthesia that exceeds 30 minutes duration to detect occurrence of inadvertent hypothermia or hyperthermia; consequently, adverse complications related to thermal disturbance may be prevented [10].

Core temperature measurement should reflect temperature of the organs that are highly perfused such as the brain or myocardium. Stone et al. [11] demonstrated that the nasopharynx, distal esophagus, and pulmonary artery are the most reliable sites to monitor intraoperative core temperature. Core temperature measurement at the pulmonary artery is the most accurate method in clinical setting, however, it is an invasive procedure. The nasopharynx and distal esophagus can be approached easily in comparison with the pulmonary artery; however, temperature probe at both sites should be carefully placed in close proximity to the internal carotid artery [12] and the lower border of the left atrium [13], respectively. In this case, we used esophageal temperature probe because of its ease of use and inexpensive disposable material property. Generally, esophageal temperature probe can be inserted into the esophageal orifice blindly at optimal depth of probe tip of $24 \mathrm{~cm}$ below the corniculate cartilages

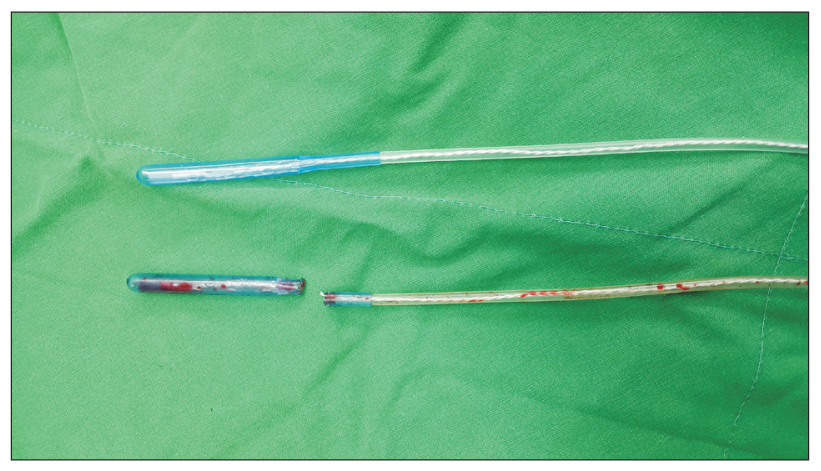

Fig. 1. The total length of the removed temperature probe was not different from a normal temperature probe. 
(lower fourth of the esophagus) in adult patient under anesthesia [14]. Usage of esophageal temperature probe is also helpful to facilitate proper auscultation of heart sound with stethoscope.

Correct placement of temperature probe is important to reflect accurate core temperature. Esophageal temperature probe should be positioned at the level of the pericardial sac to indicate myocardium temperature [15]. Due to acoustic property of esophageal temperature probe facilitating detection of heart sound, it is relatively convenient to place the probe within optimal region of contiguity between the heart and esophagus. In addition, the distal esophagus affords reliability to monitor core temperature because it is not influenced by extreme airway cooling [5]. However, malposition of the esophageal temperature probe can result in incorrect core temperature readings, and cause airway complications such as bronchospasm [8] and hypoxemia [9] due to accidental tracheal misplacement. Signs related to misplacement of probe into the trachea or bronchus include diminished heart sound and higher intra-cuff pressure requirement to seal the endotracheal tube; however, these signs may be subtle. Breathing sounds could be equal bilaterally and the healthy lung might tolerate the impediment. Thus, tracheal insertion of esophageal temperature probe may be overlooked and go undiscovered during surgery. In this case, we did not perceive sign of accidental bronchial insertion of the temperature probe because the heart sound was well heard through the esophageal stethoscope, and body temperature was judged appropriate $\left(36.2^{\circ} \mathrm{C}\right)$ without unexpectedly low temperature reading. In addition, there were no hypoxemia and intrapulmonary pressure change after probe insertion. Moreover, since the right lung was not ventilated during surgery, possibility that the temperature probe was placed in the right bronchus was overlooked.

Misplacement of esophageal temperature probe into the trachea or bronchus can impose risk of pulmonary aspiration of pharyngeal secretions or inadequate ventilation caused by imperfect cuff seal, as well as life-threatening airway problems. Therefore, assessment of proper positioning of the temperature probe is necessary to avoid these adverse events. Radiographic verification may be the most definitive method [7]; however, it is not convenient and is time consuming. Possibility of temperature probe position at the level of the trachea or bronchus may be confirmed by means of fiberoptic bronchoscopy. However, we inserted the temperature probe blindly with the patient in the left decubitus position after identification of correct position of double lumen tube through fiberoptic bronchoscopy; therefore, malposition was not suspected until the body temperature reading was interrupted and blood was observed in the temperature probe. Despite absence of signs associated with malposition of esophageal temperature probe, optimal site confirmation through fiberoptic bronchoscopy might be recommended in patient undergoing one-lung ventilation. In addition, to monitor degree of redistribution of hypothermia, insertion of temperature probe immediately after induction of anesthesia in patient under supine position may have been preferable to that after rotation to lateral decubitus position.

In conclusion, accurate insertion of esophageal temperature probe and careful assessment of correct temperature probe position is needed, especially in patients undergoing one-lung ventilation.

\section{REFERENCES}

1. Kurz A, Sessler DI, Lenhardt R. Perioperative normothermia to reduce the incidence of surgical-wound infection and shorten hospitalization. Study of Wound Infection and Temperature Group. N Engl J Med 1996; 334: 1209-15.

2. Winkler M, Akça O, Birkenberg B, Hetz H, Scheck T, Arkiliç CF, et al. Aggressive warming reduces blood loss during hip arthroplasty. Anesth Analg 2000; 91: 978-84.

3. Lenhardt R, Marker E, Goll V, Tschernich H, Kurz A, Sessler DI, et al. Mild intraoperative hypothermia prolongs postanesthetic recovery. Anesthesiology 1997; 87: 1318-23.

4. Insler SR, Sessler DI. Perioperative thermoregulation and temperature monitoring. Anesthesiol Clin 2006; 24: 823-37.

5. Kaufman RD. Relationship between esophageal temperature gradient and heart and lung sounds heard by esophageal stethoscope. Anesth Analg 1987; 66: 1046-8.

6. Liu SK, Chiang YY, Poon KS, Wen YR, Li CY, Liu YF, et al. Thoracotomy for lung lesion does not affect the accuracy of esophageal temperature. Acta Anaesthesiol Taiwan 2013; 51: 116-9.

7. Sanders JC. Deep positioning of an esophageal temperature probe may lead to overestimation of core body temperature during laparoscopic Nissen fundoplication in infants. Paediatr Anaesth 2005; 15: 351-2.

8. Stein B, Mack PF. Bronchospasm due to malpositioned esophageal temperature probe. Anesth Analg 2003; 97: 920-1.

9. Pickard WA, Reid L. Hypoxia caused by an esophageal stetho- 
scope. Anesthesiology 1986; 65: 534-6.

10. Sessler DI. Temperature monitoring and perioperative thermoregulation. Anesthesiology 2008; 109: 318-38.

11. Stone JG, Young WL, Smith CR, Solomon RA, Wald A, Ostapkovich N, et al. Do standard monitoring sites reflect true brain temperature when profound hypothermia is rapidly induced and reversed? Anesthesiology 1995; 82: 344-51.

12. Lee J, Lim H, Son KG, Ko S. Optimal nasopharyngeal temperature probe placement. Anesth Analg 2014; 119: 875-9.
13. Mekjavic IB, Morrison JB, Brengelmann GL. Construction and position verification of a thermocouple esophageal temperature probe. IEEE Trans Biomed Eng 1984; 31: 486-8.

14. Whitby JD, Dunkin LJ. Temperature differences in the oesophagus. The effects of intubation and ventilation. Br J Anaesth 1969; 41: 615-8.

15. Mekjavić IB, Rempel ME. Determination of esophageal probe insertion length based on standing and sitting height. J Appl Physiol (1985) 1990; 69: 376-9. 